\title{
Global Progressive Disease in Skin
}

National Cancer Institute

\section{Source}

National Cancer Institute. Global Progressive Disease in Skin. NCI Thesaurus. Code C159955.

25 percent or greater increase in skin disease from baseline; or new tumors (T3) in patients with T1, T2, or T4 only skin disease; or loss of response: in those with complete or partial response, increase of skin score of greater than the sum of nadir plus 50 percent baseline score. 